Revue

de Sémantique

et Pragmatique
Revue de Sémantique et Pragmatique

35-36 | 2015

Interface sémantique/morphologie

\title{
Sens morphologiquement construit et procédés concurrents : les noms de spécialistes en -logue et - logiste
}

Fiammetta Namer et Florence Villoing

\section{(2) OpenEdition \\ Journals}

Édition électronique

URL : http://journals.openedition.org/rsp/1377

DOI : $10.4000 /$ rsp. 1377

ISSN : 2610-4377

Éditeur

Presses universitaires d'Orléans

Édition imprimée

Date de publication : 1 mars 2015

Pagination : 7-26

ISSN : 1285-4093

\section{Référence électronique}

Fiammetta Namer et Florence Villoing, "Sens morphologiquement construit et procédés concurrents les noms de spécialistes en -logue et -logiste », Revue de Sémantique et Pragmatique [En ligne], 35-36 | 2015, mis en ligne le 01 mars 2016, consulté le 09 mai 2020. URL : http://journals.openedition.org/ rsp/1377; DOI : https://doi.org/10.4000/rsp.1377 


\section{SENS MORPHOLOGIQUEMENT CONSTRUIT ET PROCÉDÉS CONCURRENTS : LES NOMS DE SPÉCIALISTES EN - LOGUE ET - LOGISTE}

Fiammetta Namer, UMR 7118 ATILF-Université de Lorraine Florence Villoing, UMR 7114 MoDyCo-Université de Paris 10

\section{INTRODUCTION}

Nous nous proposons dans ce travail d'étudier les noms d'humains comme pneumologue, ou radiologiste, qui donnent à voir la séquence <logue $>$ ou $<$ logiste $>$. Nous faisons l'hypothèse que $<$ logue $>$ et $<$ logiste $>$ constituent les exposants de deux règles morphologiques construisant les noms dont nous symbolisons la structure par Xlogue et Xlogiste. La ressemblance formelle de ces deux séquences conduit à supposer, dans un premier temps, que Xlogue et Xlogiste, quand ils ne sont pas synonymes (e.g. radiologue et radiologiste), possèdent les mêmes propriétés sémantico-référentielles : les deux types de noms en effet renvoient $a$ priori à des spécialistes de ce à quoi réfère $\mathrm{X}$. Ce n'est, cependant, qu'un examen attentif des données, qu'elles soient issues d'un dictionnaire $\left(\mathrm{TLF}^{1}\right)$ ou extraites de corpus ( $\mathrm{frWaC}$ ), qui nous amènera à décider si Xlogue et Xlogiste désignent le même type sémantique d'entités : nous verrons que cette étude reviendra à débattre du statut de $<$ logue $>$ et $<$ logiste $>$, et donc à mesurer le rôle joué par la morphologie dans le calcul des propriétés sémantiques du lexique.

\footnotetext{
${ }^{1}$ Trésor de la Langue Française, cf. http://atilf.atilf.fr
} 


\section{FORMATION DE NOMS DE SPÉCIALISTES ET PROCÉDÉS RIVAUX}

Une question au coeur de l'interface morphologie/sémantique concerne la compétition entre procédés morphologiques : deux procédés vérifiant des schémas constructionnels comparables et sélectionnant les mêmes bases produisent-ils des construits synonymes ? Si oui, comment expliquer cette synonymie? Si non, qu'est ce qui les distingue ? Nous allons voir que la valeur sémantique des noms de spécialistes en $<$ logue $>$ et $<$ logiste $>$ se reflète dans leur construction morphologique, fournissant ainsi un argument supplémentaire au lien intrinsèque entre sémantique et morphologie.

\subsection{LES NOMS DE SPÉCIALISTES MORPHOLOGIQUEMENT CONSTRUITS}

Le français dispose de différents moyens morphologiques pour former des noms de spécialistes. On relève essentiellement des noms issus de dérivation, comme les suffixés en -iste, e.g. chimiste (Roché, 2011 ; Cartoni et Namer 2012) ou en -ien, e.g. mathématicien (Lignon 2002), et des composés dits néoclassiques (Namer \& Villoing 2006), dont les composants sont souvent d'origine latine ou grecque, et dont le second constituant dénote à l'origine l'activité qui caractérise le spécialiste : écrire (graph : géographe), parler (lego : morphologue), ou bien l'hyperonyme du nom : iatros dénote un médecin (pédiatre), nomos (astronome) réfère au titulaire d'une fonction. Les noms de spécialistes résultent évidemment aussi de règles de composition standard (garde-barrière), dont les constituants peuvent être eux-mêmes morphologiquement contruits (archéologue-historien); sur la question de la composition en français, voir Villoing (2012). Enfin, il existe des noms de spécialistes formés au moyen de la séquence $<$ logiste $>$ (laryngologiste) ce dont il va être question ici.

\subsection{RIVALITÉ : ALTERNANCE OU CONCURRENCE}

Deux procédés morphologiques sont considérés comme rivaux (suivant la terminologie empruntée à Plag, 1999) quand ils sélectionnent le même type de base et forment des construits possédant des propriétés sémantico-référentielles proches. L'un des indices de rivalité entre procédés est leur faculté à former ce que nous appellerons des doublons, c'est à dire des construits partageant la même base ; c'est par exemple le cas des règles de suffixation formant des noms de propriété désadjectivaux (sensibilité/sensiblerie) et de celles produisant des noms déverbaux de procès (battage/battement). Parmi les procédés rivaux, Plag 1999 distingue les cas d'alternance et les cas de concurrence. Pour les premiers, des contraintes spécifiques pèsent sur le construit (ainsi, cuisinier et cuisiniste désignent des référents différents) ou sur la base : par exemple, la contrainte de dissimilation conduit à préférer sarkozifier (ou mathématicien) à ?sarkosyzer (ou mathématiciste), où subsiste la consécution de deux phonèmes 
identiques (sur ces questions, voir Lignon 2008, Lignon \& Plénat 2009, Koehl 2012, Lignon 2013, Namer 2013).

Contrairement aux procédés en alternance, les règles concurrentes sont susceptibles de produire des construits synonymes absolus ou propositionnels, suivant la terminologie empruntée à Cruse (2004 :154-157). L'auteur propose en effet une tripartition des synonymes où il distingue les synonymes absolus, les synonymes propositionnels et les quasi-synonymes. Les premiers, très rares, partagent tous leurs traits sémantiques, et s'emploient dans le même contexte (artérien et artériel); les seconds diffèrent en terme d'expressivité, registre de langue, etc. mais renvoient exactement au même référent (cinéma et cinoche) ; enfin, les derniers partagent une partie de leurs traits sémantiques, mais diffèrent en termes de restrictions de sélection stylistique, conceptuelle et contextuelle, et ne sont pas librement substituables dans tous les contextes (démarrer et commencer).

Des exemples comme laryngologue et laryngologiste font penser qu'à première vue les doublons de noms en <logue> et en <logiste> sont des synonymes absolus, ce qui indiquerait que les règles dont ces noms sont issus sont concurrentes. C'est ce que nous allons vérifier.

\subsection{DONNÉES ANALYSÉES}

Les noms que nous avons étudiés proviennent de deux ressources complémentaires. Nous avons d'une part extrait l'ensemble des formes nominales se terminant par l'une des séquences $<$ logue $>$ et $<$ logiste $>$ du TLF. Ce premier ensemble de 297 noms a été complété par 964 autres formes munies des mêmes séquences et issues du corpus majoritairement francophone frWaC (Baroni \& al., 2009).

\begin{tabular}{|l|l|l|l|}
\hline & TLF & frWaC & Total \\
\hline Xlogue & 184 & 714 & 898 \\
\hline Xlogiste & 113 & 250 & 363 \\
\hline Total & 297 & 964 & 1261 \\
\hline
\end{tabular}

Tableau 1: Nombre et origine des noms en $\langle$ logue $\rangle$ et $<$ logiste $>$

Conçu dans le cadre du programme WaCky (Baroni \& al., 2006), frWaC comporte 1,6 milliard d'occurrences et réunit le contenu textuel de documents en ligne caractérisés par leur appartenance au domaine français.

Le consortium WaCky est le fruit d'une initiative visant à reproduire, dans un format exploitable par des outils d'analyse automatique de corpus, la diversité des contenus récents de la Toile dans des proportions comparables à l'original. L'objectif est de rendre l'accès à la Toile, qui constitue l'une des ressources textuelles les plus complètes existant actuellement, non tributaire 
des moteurs de recherche commerciaux. En particulier, la récupération des mots fléchis est rendue possible par l'emploi d'expressions régulières.

L'intérêt de ce deuxième ensemble de noms, issus de la Toile, est triple : parce qu'ils sont nouveaux (la constitution de frWaC date de 2009), ils complètent les données dictionnairiques (où aucun nouveau nom en $<$ logue $>$ ou en <logiste> n'apparaît après 2000) ; parce qu'ils sont issus de documents textuels, leur contexte d'emploi est accessible ; enfin, parce qu'ils proviennent d'un corpus clos, ces noms peuvent donner lieu à des calculs lexicométriques, comme les mesures de fréquence, ou l'évaluation de la productivité des patrons morphologiques qui les ont formés (Dal et al 2008 ; Dal et Namer 2012, à paraître).

La distribution des noms en <logue> et des noms en <logiste> laisse entrevoir des premiers résultats (cf. tableau 1) : sans surprise, tout d'abord, les néologismes trouvés dans frWaC sont trois fois plus nombreux que les noms enregistrés dans le TLF. Cet écart avoisine un facteur 7 (7 fois plus) si l'on ne regarde que les noms en $<$ logue $>$. Ceux-ci, par ailleurs, sont globalement trois fois plus nombreux que les noms en <logiste> : c'est dans le frWaC que la différence est la plus marquée, le contenu du dictionnaire pouvant laisser entendre que les deux séries de noms sont quantitativement équivalentes.

Puisque la question posée dans ce travail a trait à l'éventuelle concurrence entre ce que nous considérons être des modes de formation en $<$ logue $>$ et en $<$ logiste>, l'analyse des données procède en deux temps : notre première enquête consiste à examiner les doublons formés par ces deux patrons. Ensuite, nous nous intéressons à l'interprétation des singletons, de manière à faire émerger les similitudes et les différences entre ces deux modes de formation.

\section{INTERPRÉTATION DES RÉSULTATS}

\subsection{DOUBLONS}

Dans l'ensemble des noms provenant du TLF + frWaC, on relève 147 doublons (sur 1261); en d'autres termes, 40\% des Xlogiste et 13,5\% des Xlogue partagent le même radical X. Seuls $28 \%$ des couples (Xlogue, Xlogiste) sont intégralement issus de $\mathrm{frWaC}$ (indologue, indologiste), alors que, dans $45 \%$ des cas, les deux membres du couple appartiennent au TLF (ophtalmologue, ophtalmologiste).

$80 \%$ de l'ensemble des doublons sont qualifiables de synonymes absolus au sens de Cruse (2004), e.g. pathologue et pathologiste. La différence entre les deux formes porte sur :

- la date de première apparition (ainsi, éthologiste est plus récent qu'éthologue), 
- les fréquences d'emploi (par exemple Google relève 16.100 occurrences de gynécologiste, contre plus de 4 millions de gynécologue)

- l'entrée en désuétude de l'une d'elles (selon le TLF, biologue aurait été abandonné au profit de biologiste),

- ou, enfin, son origine, les Xlogiste étant souvent des calques ou des emprunts de l'anglais (comme e.g. phraséologiste, contrairement à phraséologue).

Parmi les $20 \%$ de doublons restants, certains correspondent à ce que Cruse 2004 nomme des quasi-synonymes : ainsi, d'après le TLF, le vexillologue est un spécialiste des drapeaux, alors que le vexillologiste les collectionne, et si le gérontologue est un médecin, le gérontologiste, lui, ne l'est pas toujours. Le restant des couples (Xlogue, Xlogiste) est formé de noms possédant des sens distincts : par exemple, l'écologue est un spécialiste d'écologie alors que l'écologiste en désigne un partisan ; l'aérologue est un médecin (ce nom n'est d'ailleurs presque plus utilisé), cependant que l'expertise d'un aérologiste concerne les véhicules qui volent.

L'observation des doublons conduit aux constats suivants :

- la présence hégémonique de synonymes parmi les doublons est compatible avec la notion de concurrence,

- les doublons sont cependant minoritaires, puisque $60 \%$ des Xlogiste et $86,5 \%$ des Xlogue en sont privés

- les doublons s'observent principalement avec les données du TLF, et ne fournissent pas d'indication sur le comportement des noms étudiés dans le lexique attesté.

Pour confirmer ou démentir la concurrence supposée des patrons en $<$ logue $>$ et $<$ logiste $>$, il nous faut donc examiner les Xlogue et Xlogiste singletons, du TLF et du frWaC, afin de déterminer s'ils partagent les mêmes propriétés sémantico-référentielles.

\subsection{NOMS EN <LOGISTE >}

Qu'ils soient enregistrés ou néologiques, singletons ou en doublon avec Xlogue, les Xlogiste renvoient de façon régulière à des humains spécialistes dans un champ relevant surtout des sciences formelles, physiques ou naturelles (1), même si parfois le référent peut être professionnellement relié à une discipline des sciences humaines et sociales ou littéraires (2). Très souvent, Xlogiste nomme un scientifique ou un praticien dans un domaine (bio-)médical ou paramédical (3).

(1) gemmologiste, minéralogiste

(2) phénoménologiste, démonologiste

(3) sérologiste, néonatalogiste 
Parfois Xlogiste désigne un professionnel dont les diplômes et la compétence le distinguent du simple amateur dans un domaine technique donné :

(4) aquariologiste (versus aquariophile)

À de rares occasions, la création de Xlogiste semble exprimer également de la part du locuteur sa dérision vis-à-vis de celui dont il parle (5a, 5b)

(5) a car Bayrou est un Bayroulogiste centriste croyant, non-pratiquant

b Au fil de tant d'impostures, Daniel Cohn-Bendit ne serait qu'un téléécologiste, représentant d'un dernier avatar du libéralisme

\subsection{NOMS EN <LOGUE>}

Les noms en $<$ logue $>$ issus du TLF ont un comportement assez semblable à celui des Xlogiste. Ils servent en effet à nommer les spécialistes, praticiens ou chercheurs, dans les domaines du (para)médical en particulier et des sciences et techniques en général (6).

(6) pneumologue, climatologue, planétologue

Cette tendance se retrouve avec les néologismes trouvés dans frWaC, où les noms en Xlogue continuent à répondre à ce besoin conceptuel qu'est l'acte de nommer un nouveau référent humain, reconnu pour son expertise dans une spécialité médicale (7) ou paramédicale, éventuellement non réglementée (8); la création de noms en $<$ logue $>$ permet de cette manière d'identifier de nouveaux spécialistes, qui s'occupent de branches particulières dans les disciplines scientifiques officielles. Ainsi, sous (9), la spécialité de l'individu dénoté relève, respectivement, de l'optique, la médecine, la météorologie, la botanique, l'anthropologie, et la stomatologie.

(7) hématologue, herpétologue

(8) réflexologue, relaxologue ( En séance individuelle ou en groupe, le rôle du relaxologue est d'accompagner la personne dans sa connaissance d'elle-même, afin de l'aider à gérer son stress et ses angoisses, en s'adaptant à ses besoins spécifiques par un suivi personnalisé »)

(9) contactologue, lacrymologue, nivologue, orchidologue, raciologue, implantologue

L'éventail des types d'agents humains auxquels un nom en Xlogue permet de référer inclut également les experts dans des disciplines émanant des sciences humaines et sociales : histoire, économie, gestion, géographie, religion (10a), de l'art, la littérature et le sport (10b), des départements recherche et développement du commerce et de l'industrie (10c) ainsi que des domaines farfelus (la science des ovnis) voire contestables (certaines 'médecines' alternatives, la voyance) (10d) :

(10) a khmérologue, japonologue, islamologue, éventrologue ( < Jack l'Eventreur)

b lupinologue (< Arsène Lupin), célinologue, organologue, rugbyologue 
c caféologue ('vérifie la qualité des grains de café'), cosmétologue ('scientifique chargé de mener et/ou de participer aux phases d'étude, d'analyse, d'élaboration et d'évolution de produits cosmétiques')

d ovnilogue, humanologue, tarologue, hypnologue, arithmologue, chirologue

Outre la dénomination d'experts dans un domaine de connaissance plus ou moins légitime, on voit émerger avec les noms en Xlogue de nouvelles catégories référentielles. Ainsi, le référent du Xlogue peut être non pas celui qui connaît X, mais celui qui en est l'auteur (11), le créateur (12), ou encore celui dont la finalité est l'obtention de ce à quoi renvoie X (13).

(11) rumorologue ('auteur d'une rumeur')

(12) bruitologue ('créateur de bruits/bruitages')

(13) licenciologue ('consultant chargé de préparer les plans de licenciements')

Le nom Xlogue peut également dénoter l'amateur, le collectionneur passionné, obsédé de ce à quoi $\mathrm{X}$ réfère :

(14) pochettologue (< pochettes de disques), tégestologue (< sous-bocks de bière), bédélogue, chouettologue, cyclonologue, jardinologue, pharologue

Enfin, les acteurs du monde des médias audiovisuels et de la stratégie en marketing politique, en définissant les domaines sociétaux qu'ils donnent à voir au public comme prioritaires, sont à l'origine des Xlogue destinés à nommer les analystes et autres experts de ces domaines : la notoriété du nom en <logue> croît alors avec celle de l'impact médiatique que ces mêmes journalistes font gagner au domaine qu'ils traitent, et auquel renvoie $X$ (15). Il peut s'agir d'un conflit (a), d'une campagne de prévention sanitaire (b), de questions de société (c), de prévisions économiques (d), d'élections (e), de politique intérieure (f) ou internationale (g).

(15) a polémologue ('géopoliticien spécialiste des conflits')

b tabacologue, accidentologue, préventologue (< prévention des risques),

c audimatologue, médiaphobologue

d déclinologue, nihilistologue

e sondologue, tendançologue ('analyse en direct les tendances électorales')

f solférinologue ( $<$ rue de solférino), conacologue ${ }^{2}$

g violentologue ('nom donné en Colombie aux analystes de la violence')

L'examen des données issues de frWaC montre que la création de néologismes en <logue $>$ répond, chez son auteur, à d'autres motivations que la nécessité de nommer de nouveaux référents. En effet, Xlogue possède parfois une capacité référentielle identique à celle d'un nom préexistant, et satisfait ici un besoin énonciatif, qu'il soit d'ordre diatopique, comme les variantes québecoises

${ }^{2}$ Nom utilisé le 3/10/13 par T. Legrand sur France Inter pour désigner les membres du gouvernement socialiste après la série de 'couacs' à propos du clash Valls-Duflot. 
en (16), ou diaphasique. En (17), l'emploi des noms en <logue> confèrent, aux yeux du scripteur, plus de prestige à la fonction, respectivement, de sage-femme ou garagiste. A l'inverse, en (18), l'utilisation de Xlogue peut comporter une fonction hypocoristique, et permettre au scripteur de gommer la distance qui le séparerait sinon du spécialiste qu'il nomme (ainsi l'arbrologue s'occupe de généalogie). La relation qui unit le scripteur et l'individu dénoté est généralement celle de patient-thérapeute : le rapprochement traduit l'affection et la familiarité que le premier ressent pour le second (18a) et/ou atténue par la légèreté de ton la gravité médicale à laquelle on pourrait associer la spécialité (18b).

(16) denturologue ('orthodontiste'), urgentologue ('urgentiste')

(17) parturologue ('sage-femme'), pneumaticologue, radiatorologue ('garagiste')

(18) a bébélogue

b cervologue, dingologue, migrainologue

Très souvent, la création de Xlogue répond, pour son auteur, à un besoin à la fois conceptuel et énonciatif : cet auteur invente un nom de spécialiste envers lequel il émet un jugement. Comme l'indiquent les contextes ou définitions donnés entre parentèses en (19-20), cette opinion peut être négative quand l'individu nommé est un escroc, qu'il usurpe la spécialité dont la véritable autorité échoit à quelqu'un d'autre, nommé autrement (19). Le sentiment de mépris peut également être dirigé contre la (pseudo) spécialité de l'individu incriminé que dénote Xlogue (20).

(19) pédagologue (« le pédagologue est celui qui parle de pédagogie sans la pratique »), castellologue ('archéologue du dimanche'), négrologue ('qui se croit spécialiste de la culture africaine')

(20) pseudologue (« Donc Heidegger est un sophiste, et ce qu'il enseigne, faux. Comme enseigner des faussetés est [...] le fait d'un pseudologue »), expertologue («A chaque année son bobardeur pédant : voici le tour de Philippe Manière, 'young leader' de la French-American Foundation et expertologue cumulard de la presse écrite et radio »)

rumorologue, trucologue ( Ce qui permet aussi, aux toubibs, spécialistes et autres trucologues de cardiologie, de boyaulogie, d'exercer leur art majestueusement, pédamment et plein de rapport ; chacun se fichant comme d'une guigne de ses collègues pneumologues ou urologues »),

bobologue (" le médecin généraliste est un docteur bobologue »), champixologue ('promoteurs du Champix, médicament que les associations jugent dangereux'),

cyclistologue, alibitologue ('qui vend ses excuses farfelues aux maris infidèles'), peoplelogue (" Quand à nos 2 néo-cons politologueéconomicologue-peoplelogue ... »).

Lorsque l'invention de Xlogue n'a pas de connotation péjorative, alors elle répond à un désir du scripteur de faire de l'humour, en créant un nom 
de spécialiste futile ou fantaisiste (21), voire oxymorique (22). Le contexte d'apparition de ces formes, ainsi que leurs fréquences peu élevées, indiquent qu'il s'agit de créations éphémères, réalisées au moment de l'énonciation, servant souvent à qualifier le scripteur lui-même (23).

(21) blogologue, cévélogue, dahulogue, entonoirologue, espérologue, habillologue, voyoucratologue, radinologue, nounoursologue, poulologue

(22) machinologue, xxxlogue, toutologue

(23) espérologue («Un métier à inventer...pas facile! heu... l'espérologue.. celui qui invente l'espoir et le rêve et qui les sème dans l'air »)

habillologue ( «aisse moi deviner qui elle est, je suis habillologue (c'est à dire que je lis dans les habits ce que les gens sont).»)

voyoucratologue ('autoqualification de l'auteur d'un billet commentant l'intervention de Sarkozy sur la voyoucratie'),

radinologue ( "Ma chaudière est dans ma cave...et je suis radinologue de l'éclairage... »)

Portée à son comble, la volonté du scripteur de faire de l'humour et de se démarquer se traduit dans la construction d'occasionnalismes en <logue > par le biais de formations en rafales, dont le but est moins de véhiculer un message que de faire des rimes. Ce phénomène d'entraînement, observé fréquemment avec Xlogue (24) a été par ailleurs remarqué dans de nombreux travaux de morphologie portant sur les données de la Toile, et semble caractériser singulièrement cette ressource (cf. entre autres Dal et al 2004 ; Dal \& Namer 2012 ; Lignon 2013 ; Lignon \& Namer 2010, 2013 ; Koehl 2012 ; Namer 2013 ; Tanguy 2012).

(24) « Explication de l'oisologue, volatilologue, plumologue, volaillologue, galinacéologue, piafologue, le professeur Erick BERNARD »

«Tout le monde a une spécialité : moi je suis «c'est-pas-gravologue». Et vous, quelle est la vôtre ? minçe-alorologue.........spécialiste des sourcils relevés d'étonnement.......... : ) - Positive attutidologue cela reclame beaucoup d'effort mais l'on se sent rarement mal - Moi c'est plutôt «Comment-je-vaism'en-sortirologue»!»

« C'est quoi un pédagologue ?? -- Il répare les pédalos, qui naviguent sur nos beaux cours d'eaux ?? -- Suis-je alors un cheminologue ?? -- Quitte à choisir, je préfère être un humourologue, mieux vaut le prendre comme ça. -- Si seulement je pouvais être éclairé par un éclairologue...»

«À la demande personnelle de Thomas, nous recherchons UNE poulologue qui serait également poussinologue et coqologue»

\subsection{BILAN}

L'observation des données conduit à différents constats : les Xlogue et Xlogiste enregistrés dans le TLF dénotent des spécialistes ou praticiens de domaines scientifiques, plus particulièrement médicaux. Nous n'avons trouvé aucun indice déterminant en faveur de l'hégémonie de l'un ou l'autre type de 
nom : comme en témoignent les rubriques étymologiques illustrées ci-dessous, le TLF signale en effet tantôt l'abandon de formes en Xlogue au profit de leur doublon en Xlogiste (25), tantôt la situation inverse (26).

(25) «Il semble qu'éthologiste soit devenu le terme le plus usuel pour désigner le spécialiste de l'éthologie animale » (cf. Lar. encyclop. Suppl. 1968 et Lar. Lang. fr.). (s.v. éthologue)

(26) « A remplacé vulcanologiste »(1910, Lar. mens., loc. cit.), empr. à l'angl. vulcanologist (dep. 1858, MALLET ds NED) (s.v. vulcanologue)

Mais cette apparente homogénéité sémantico-référentielle de Xlogue et Xlogiste, qui apparaît dans les données anciennes où les deux sont interchangeables ${ }^{3}$ est contredite par l'examen des noms en $<$ logue $>$ et $<$ logiste $>$ présents dans les textes réunis dans le corpus frWaC, dont les variations diaphasiques, diastratiques et diatopiques (suivant par exemple la répartition de Labov 1976) du contenu garantit une projection quantitativement et qualitativement raisonnable de l'usage avéré des formes étudiées.

En effet, dans le lexique que l'on peut qualifier d'authentique, par opposition au lexique enregistré que constituent les entrées du TLF, les Xlogue et Xlogiste montrent des différences notables dans les types sémantiques des référents identifiés.

D'une part, les locuteurs (ou plutôt : les scripteurs) de la langue générale semblent s'être approprié le mode de formation en <logue> pour nommer des individus possédant un certain niveau d'expertise dans un domaine plus ou moins spécialisé et parfois fantaisiste ; de l'autre, les noms en <logiste> continuent à relever de technolectes où ils restent cantonnés dans la dénotation de spécialistes.

Nous pouvons donc affirmer qu'en synchronie, le procédé de construction de noms en $<$ logue $>$ et celui en <logiste $>$ ne servent pas à former le même type de noms. Les référents des Xlogue et Xlogiste sont certes des humains identifiés par leur expertise dans un domaine donné ; cependant, si les noms en Xlogiste sont de nature homogène, ceux en Xlogue, en revanche, entretiennent quasiment n'importe quelle relation (connaisseur, auteur, amateur,...) avec le référent de $\mathrm{X}$. La différence sémantique entre Xlogue et Xlogiste reflète celle que l'on perçoit dans l'intention des locuteurs qui emploient ces noms : la création et l'utilisation de Xlogiste répond à un besoin conceptuel, alors qu'à cette fonction

3 Notons que cette substituabilité n'est, le plus souvent, présente que dans les dictionnaires : ainsi, Littré (1872) donne comme synonymes biologiste et biologue. Cependant, l'outil Ngram Viewer de Google Book, qui indexe à ce jour plus de 8 millions de livres (Lin et al., 2012), montre que même entre 1800 et 1900 le nombre d'occurrences de biologue a toujours été proche de 0 , contrairement à celui de biologiste. 
dénotative s'ajoute, dans le cas des noms en $<$ logue $>$, une volonté de la part du scripteur de faire de l'humour, d'exprimer sa sympathie ou d'afficher son mépris vis-à-vis du référent de Xlogue ou de celui de X.

En somme, Xlogue et Xlogiste ne sont en aucun cas des patrons de formation de noms synonymes, et $<\operatorname{logue}>$ et $<$ logiste $>$ ne peuvent prétendre au statut d'exposants formels de procédés concurrents, si l'on se reporte à la définition qui est donnée au § 2.2. Reste alors à vérifier si ces procédés constructionnels sont qualifiables de rivaux, ce dont on va pouvoir s'assurer grâce à l'analyse morphologique.

\section{ANALYSE MORPHOLOGIQUE}

Rappelons que deux procédés sont qualifiables de rivaux s'ils s'appliquent à un même type de base et produisent des construits référentiellement comparables. Si les procédés à l'origine des Xlogue et Xlogiste sont rivaux, alors cette caractéristique devrait se refléter dans l'analyse morphologique de ces deux types de noms, notamment en précisant leur parenté.

Pour vérifier cette hypothèse, nous examinons l'une après l'autre deux hypothèses de formation des Xlogue et Xlogiste. Dans cette optique, nous nous situons dans le prolongement du cadre théorique de la morphologie dite lexématique (pour un survol : Fradin 2003, pour une application récente au français : Fradin et al 2009) qui présente deux propriétés cruciales pour notre étude. L'unité est le lexème, et le procédé de construction qui manipule cette unité se manifeste sous la forme d'une relation entre base(s) et construit où interagissent trois mécanismes simultanés et indépendants : l'un agit sur la forme, le second établit le rapport catégoriel entre l'input et l'output, et le troisième se charge de définir le lexème construit en fonction du sens de sa base. Par ce choix théorique, nous nous affranchissons des biais, induits, dans les théories s'appuyant sur les courants morphématiques incrémentaux de la morphologie, par les opérations de concaténation de morphèmes déclenchées ou bloquées suivant des contraintes pesant sur la base, et assignant aux mots construit un sens compositionnel, dont la complexité croît avec le nombre de constituants formels.

Parmi les analyses possibles, il en est deux qui viennent spontanément à l'esprit, mais que nous rejetons immédiatement, pour des raisons sémantiques. La première est celle de la filiation directe Xlogue > Xlogiste, le second s'analysant par conséquent comme dérivé du premier par suffixation en -iste. La seconde conçoit /log/ et /lozist/ comme deux exposants allomorphes d'un même procédé. Nous n'exposons pas ici ces hypothèses, ni leur réfutation, dont on pourra trouver le détail du raisonnement dans (Villoing \& Namer, 2014). 


\subsection{HYPOTHÈSE 1 : ÉMERGENCE D'UN NOUVEAU MODE DE FORMATION EN $<$ LOGISTE >}

La figure 1 représente la relation que Xlogue et Xlogique se doivent d'entretenir si on considère ces schémas de noms comme émanant de procédés rivaux.

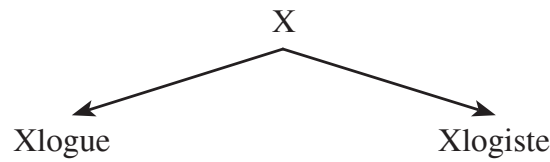

Fig 1 : Xlogue et Xlogiste formé par deux procédés rivaux

Suivant ce schéma, les deux noms ont X pour ascendant direct potentiel, ce qui rend possible l'émergence de doublons, dès lors que les deux dérivations sont simultanément activées. Cette situation se produit lorsque l'intention du locuteur est de former le nom du spécialiste d'une discipline scientifique reconnue. Il a alors librement à sa disposition la formation de Xlogue, ou celle de Xlogiste. En revanche, quand il s'agit de nommer un individu d'après son expertise (autoproclamée) ou sa passion vis-à-vis de ce que $\mathrm{X}$ dénote, seule la formation en Xlogue est disponible. Cette solution, qui repose sur l'émergence d'un nouveau mode de formation en <logiste>, a cela de séduisant qu' elle rend compte du besoin de la part du locuteur de renforcer l'interprétation de «vrai scientifique » (vs « amateur farfelu ») qu'il attribue aux noms en Xlogiste grâce à la succession des séquences $<$ logue $>$ et $<$ iste $>$, chacune d'elle étant, indépendamment de l'autre, reconnaissable comme l'exposant d'une règle formant des noms d'experts (pour -iste, cf. Roché 2011). La combinaison des deux accentue ainsi, dans le nom généré, le sérieux scientifique de l'individu dénoté.

Formellement, cette solution rappelle, sans lui correspondre totalement, un mécanisme souvent observé en morphologie flexionnelle, et connu sous le nom d'exponence multiple ou multiexponence (Baerman \& Corbett 2012) : ce nom désigne en effet le phénomène qui caractérise un mot-forme comportant plusieurs manifestations d'un même trait morphosyntaxique. L'un des exemples mentionnés dans la littérature est celui de la formation du participe passé régulier allemand en geXt (e.g. gekauft). Cependant, à la différence de l'exponence multiple, l'emploi du patron dérivationnel en <logiste > suggère par le cumul de formes (qui n'ont plus rien à voir avec des marques morphosyntaxiques) un renforcement du sens attribué au nom de sortie.

Malgré les avantages cognitifs qu'elle apporte, cette solution laisse sans réponse un certain nombre de questions : elle n'explique pas l'infériorité numérique des Xlogiste vis-à-vis des Xlogue, ni ne prédit ce qui bloque l'activation du patron en <logiste> lors de la création d'un nom désignant un « faux » spécialiste. Comme nous allons le voir, un élément de réponse à ces 
questions se trouve dans l'asymétrie du schéma constructionnel conduisant à la formation de ces deux noms, dont la figure 1 ne réussit pas à rendre compte. Pour y parvenir, nous faisons intervenir dans la structure le nom qui désigne le domaine de connaissance de X : Xlogie.

\subsection{HYPOTHÈSE 2 : XLOGUE, XLOGISTE ET LE DOMAINE D'EXPERTISE XLOGIE}

Un nom en Xlogie (comme épidémiologie ou plaisirologie) renvoie au domaine d'étude, de connaissance, de ce à quoi réfère X. La parenté de Xlogie avec Xlogue et Xlogiste est donc indiscutable, mais c'est la nature exacte du lien qui unit ces trois types de noms que la suite de cette section va permettre de déterminer. Nous allons voir que la structure paradigmatique qui en résulte est le reflet du mécanisme cognitif mis en oeuvre par les locuteurs dans leur décision de favoriser Xlogue ou Xlogiste.

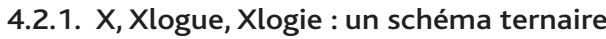

La caractéristique remarquable des formations en Xlogue (dénotant l'humain expert ou connaisseur) et en Xlogie (référant au domaine de connaissance) est qu'elles sont consubstancielles l'une à l'autre : on perçoit en effet aisément que si un individu est qualifiable d'expert ou d'amateur de $\mathrm{X}$, alors il est fort probable que le besoin de nommer l'étude de $\mathrm{X}$ émerge très rapidement. Et inversement, l'identification de X comme objet digne d'intérêt, pouvant susciter de la curiosité ou un besoin de connaissance, est indissociable de l'existence d'humains manifestant ce goût, cette curiosité. Ainsi, tout en étant chacun morphologiquement affilié à X (dès lors que X est toujours accessible au locuteur) les noms en Xlogue et Xlogie sont mutuellement dépendant l'un de l'autre, formellement et sémantiquement, pour leur formation, comme le montre, dans la figure 2, la double définition à laquelle chacun de ces deux types de noms peut être associé.

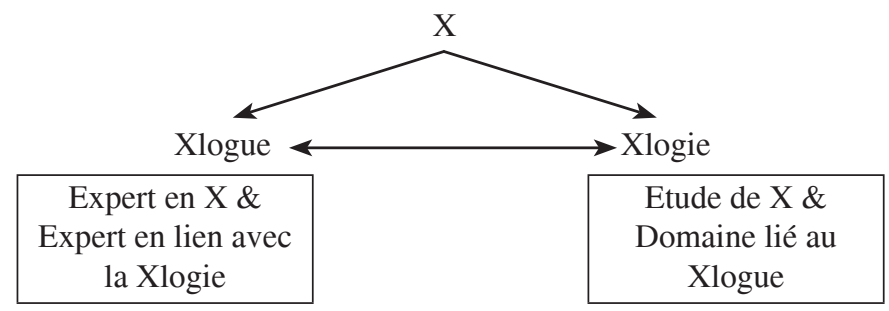

Fig 2: Schéma ternaire (X, Xlogue, Xlogie)

Cette double parenté n'est pas sans rappeler celle qui relie le nom en Xisme, dénotant une idéologie ou un courant de pensée (marxisme), le nom en Xiste, faisant référence à l'adepte de cette idéologie (marxiste), et $\mathrm{X}$, le nom de l'entité valorisée (Marx). Pour une analyse détaillée des noms en -isme, nous renvoyons à Roché (2011) qui revendique ce que nous pouvons qualifier 
d'approche paradigmatique des relations dérivationnelles. Comme l'illustre la figure 2, le schéma de construction réunissant les lexèmes X, Xlogue et Xlogie est organisé en une famille où les liens de parenté peuvent être non orientés (ce qu'indique la double flèche) : le locuteur reconstruit Xlogue à partir de Xlogie et vice-versa.

Dans cette famille dérivationnelle (cf. tableau 2), l'ensemble des membres peuvent être instanciés en synchronie (1), ou un parent peut faire défaut ${ }^{4}$ : soit parce qu'il n'a encore jamais été employé (comme radinologie en (2a) ou ultrasonologue en (2b)), soit parce que son sens est (devenu) peu voire non accessible. Ainsi, tous les locuteurs ne vont pas reconnaître immédiatement dans la séquence /pãd/ en (3a) la base pendule, qui renvoie à l'instrument employé par le pendologue pour faire de la pendologie. Ils seront encore moins nombreux, en (3b), à identifier dans échinologue « spécialiste des oursins » (respectivement dans molysmologue "spécialiste de pollution ») la base « oursin » (respectivement dans « tache, souillure ») réalisée sous la forme du radical /ekin/ (resp. /molism/), tous deux issus du grec. Enfin, plus rarement, on assiste à l'inactivation de deux sommets du triangle. En (4), Xlogie n'existe pas (encore) et le radical de X permet difficilement de retrouver cette base : la séquence /upopo/, mise pour " Ouvroir de Politique Potentielle » et forgée par analogie sur Oulipo, ainsi que /damist/, radical de dame (le damistologue joue aux dames) et non pas de damiste, sont difficilement interprétables (sur les phénomènes de décalage entre le sens et la forme d'une base constructionnelle, cf Hathout \& Namer, 2014).

\begin{tabular}{|l|l|l|l|}
\cline { 2 - 4 } \multicolumn{1}{l|}{} & X & Xlogue & Xlogie \\
\hline 1 & allergie, bébé, dahu & $\begin{array}{l}\text { allergologue, bébéologue } \\
\text { dahuologue }\end{array}$ & $\begin{array}{l}\text { allergologie, bébéologie, } \\
\text { dahuologie }\end{array}$ \\
\hline 2a & radin(erie) & radinologue & - \\
\hline 2b & ultrason & - & ultrasonologie \\
\hline $3 \mathrm{a}$ & /pãd/ (pendule) & pendologue & pendologie \\
\hline $3 \mathrm{~b}$ & $\begin{array}{l}\text { /ekin/ } \\
\text { /molism/ }\end{array}$ & $\begin{array}{l}\text { échinologue } \\
\text { molysmologue }\end{array}$ & $\begin{array}{l}\text { échinologie } \\
\text { molysmologie }\end{array}$ \\
\hline 4 & $\begin{array}{l}\text { /upopo/ } \\
\text { /damist/ }\end{array}$ & $\begin{array}{l}\text { oupopologue } \\
\text { damistologue }\end{array}$ & - \\
\hline
\end{tabular}

Tableau 2 : Réalisations possibles des sommets X, Xlogue et Xlogie

L'approche illustrée ici, dont une ébauche, sous le terme de "patrons cumulatifs », est présente dans Bochner (1993), est mentionnée chez différents partisans d'une «Word-based morphology », cf. entre autres Haspelmath (2002),

${ }^{4}$ Les (absences d')attestations répondent à des requêtes formulées via le moteur de recherche Google courant juin 2013. 
Plag (2003) ou encore Booij (2010) dont la théorie s'inspire des Grammaires de Constructions. Enfin, Hathout (2009) développe un modèle théorique visant à proposer une analyse globale du lexique, où les relations morphologiques portant sur des données authentiques croisent les notions de proximité, de familles et de séries et génèrent des paradigmes à partir de mécanismes d'analogie formelle. On trouvera dans Hathout (2011) une application de ce modèle, centrée sur l'analyse de la préfixation en anti-.

\subsubsection{Le Xlogiste : un spécialiste de Xlogie}

Contrairement à celle des Xlogue, la formation des Xlogiste n'est pas en relation de dépendance mutuelle avec Xlogie. En effet, l'observation de l'intégralité des noms en <logiste> (qu'ils soient enregistrés dans le TLF ou attestés dans frWaC) conduit au constat suivant : l'existence d'un Xlogiste est tributaire de celle du Xlogie apparenté. En d'autres termes, Xlogiste constitue la sortie de la règle de dérivation en -iste appliquée à Xlogie, ce dont rend compte la figure 3, qui complète la figure 2 par l'insertion du dérivé Xlogiste de Xlogie.

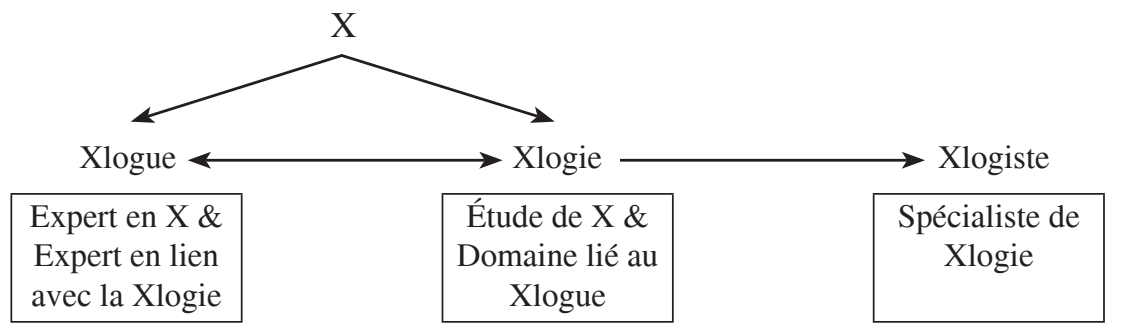

Fig 3: Schéma ternaire (X, Xlogue, Xlogie)

En conséquence (cf. tableau 3), l'existence de Xlogiste (« spécialiste de Xlogie ») implique nécessairement celle de Xlogie (ce que nous avons pu vérifier dans le corpus) (1), indépendamment de celle de Xlogue (2), et de la compréhension que le locuteur a de X (3), ce qui revient à dire que l'absence de Xlogie entraîne celle de Xlogiste (4). Bien entendu, l'inverse est faux : Xlogie peut être attesté sans que Xlogiste ne le soit (5).

\begin{tabular}{|l|l|l|l|l|}
\hline & X & Xlogue & Xlogie & Xlogiste \\
\hline 1 & $\begin{array}{l}\text { algue } \\
\text { planète }\end{array}$ & $\begin{array}{l}\text { algologue } \\
\text { planétologue }\end{array}$ & $\begin{array}{l}\text { algologie, } \\
\text { planétologie }\end{array}$ & $\begin{array}{l}\text { algologiste, } \\
\text { planétologiste }\end{array}$ \\
\hline 2 & ultrason & - & ultrasonologie & ultrasonologiste \\
\hline 3 & /ekin/ & échinologue & échinologie & échinologiste \\
\hline 4 & $\begin{array}{l}\text { entonoir } \\
\text { /damist/ }\end{array}$ & $\begin{array}{l}\text { entonoirologue } \\
\text { damistologue }\end{array}$ & - & - \\
\hline 5 & glace & glaçologue & glaçologie & - \\
\hline
\end{tabular}

Tableau 3 : Famille X, Xlogue, Xlogie, Xlogiste 
L'analyse morphologique schématisée dans la figure 3 rend compte des propriétés sémantiques relevées dans la section 3. D'une part, l'éventuelle synonymie entre Xlogue et Xlogiste, quand ce doublon existe, met en jeu la reconnaissance de Xlogie comme domaine scientifique qui répond à un besoin de théorisation de ce à quoi $\mathrm{X}$ réfère (ainsi, le planétologiste est le spécialiste de la planétologie, champ disciplinaire scientifiquement reconnu et en lien avec le planétologue qui, lui, est le spécialiste des planètes). D'autre part, cette même analyse permet de rendre compte, par l'asymétrie constructionnelle entre Xlogue et Xlogiste, du déséquilibre numérique existant entre ces deux types de noms d'experts. Corrélativement, on comprend comment Xlogue soit devenu le qualificatif attribué à tout amateur ayant une (vague) compétence en ce que dénote $\mathrm{X}$. Enfin, on peut prédire les conditions dans lesquelles un nouveau Xlogiste pourra être forgé, en examinant le comportement de Xlogie. Prenons l'exemple (5) du tableau 3, et les contextes en $(27,28)$ : le glaçologue se targue non seulement de fabriquer des glaces, mais également d'en dériver un concept qu'il nomme glaçologie. Cependant, tant que ce concept ne sera pas reconnu comme un domaine d'expertise nécessitant l'intervention de spécialistes, on ne rencontrera pas de glaçologistes.

(27) « concours : Mettez vous aux parfums. Le glaçologue français qui aura su convaincre les experts grâce à sa création glacée originale et savoureuse partira au mois de novembre à Saint Domingue »

(28) «La glaçologie Quèsaco ? Ne cherchez pas dans le dictionnaire, ce mot a été inventé par Ben and Jerry's pour l'occasion. Sachez jeunes gens que c'est l'art de faire des glaces soi-même. Au programme, petits fours, discussion avec Ben et Jerry, s'il vous plait, cours de glaçologie et Blind test où il faut deviner les goûts des glaces que les hôtes présentent aux invités. »

\section{CONCLUSION}

Nous avons étudié deux séries de noms morphologiquement apparentés, réputés désigner des spécialistes : les Xlogue et les Xlogiste. L'observation en corpus de la valeur référentielle de ces noms montre que chacun de ces deux types constructionnels a évolué différemment. Cette différence sémantique est à mettre en rapport avec un troisième type de nom, Xlogie, membre de la famille morphologique de Xlogue et Xlogiste.

En synchronie Xlogiste dénote exclusivement le spécialiste de la Xlogie. La présence, dans la structure de Xlogiste, de deux segments évoquant l'« expert» (/log/ et /ist/) semble révéler la volonté, de la part du scripteur, de consacrer à l'humain dénoté par ce type de nom la condition de spécialiste (voire d'hyperspécialiste).

Certes, parfois Xlogiste et Xlogue sont synonymes, et Xlogue dénote alors lui aussi un spécialiste. Mais le plus souvent Xlogue décrit un pseudo- 
scientifique, un amateur, collectionneur, fanatique, etc. de $X$ : la nécessité de reconnaître et nommer un expert n'est plus la priorité du locuteur quand il nomme un humain au moyen d'un nom en <logue $>$.

Nous avons vu comment l'analyse morphologique rend compte à la fois des cas de synonymie et des différences entre Xlogue et Xlogiste. Cette analyse adopte les partis pris suivants, inspirés du modèle léxématique de la morphologie et dont la vocation est descriptive, prédictive et non prescriptive : une relation morphologique, non nécessairement orientée ni exclusivement binaire, s'opère entre des lexèmes ; la construction d'un lexème n'implique pas toujours une covariation du sens et de la forme ; le point de vue du locuteur est pris en compte ; enfin, le lexique en devenir est perçu comme une projection du lexique réel existant.

Ces choix nous permettent d'envisager les procédés de construction de lexèmes sous la forme de structures paradigmatiques. Ces structures, appliquées à des données authentiques, reflètent le mécanisme en œuvre chez le locuteur : notamment, la formation et la compréhension des Xlogue et des Xlogie sont interdépendantes.

Un aspect de l'analyse morphologique n'a pas été abordé ici, et concerne le statut de $<$ logue $>$ et $<$ logie $>$ : s'agit-il d'affixes, c'est à dire d'exposant de règles de dérivation, ou d'éléments de composition, c'est-à-dire de radicaux savants de lexèmes ? Cette question, qui constitue le cas particulier d'une problématique récurrente en composition néoclassique, fera l'objet de recherches futures. 


\section{BIBLIOGRAPHIE}

Amiot D. et Dal G. (2006), "Integrating Combining Forms into a Lexeme-Based Morphology". in Booij G. et alii (eds.), MMM5 Proceedings, Fréjus : CNRS. 323336.

Baerman, M. et Corbett, G.G. (2012). "Stem alternations and multiple exponence". Word Structure 5(1) : 52-68.

Baroni, M. et Bernardini, S. (éds.) (2006), Wacky! Working papers on the Web as Corpus. Bologna : GEDIT.

Baroni M. et alii. (2009), "The WaCky Wide Web: A Collection of Very Large Linguistically Processed Web-Crawled Corpora”. Language Resources and Evaluation 43(3) : 209-226

Bochner, H. (1993), Simplicity in generative morphology. Publications in Language Sciences, vol. 37. Berlin-New York : Mouton de Gruyter.

Booij, G. (2010). Construction Morphology. Oxford : Oxford University Press.

Cartoni, B. et Namer, F. (2012). «Linguistique contrastive et morphologie : les noms en -iste dans une approche onomasiologique ». 3 CMLF, Lyon : ILF. 1245-1260.

Cruse D.A. (2004). Meaning in Language. Oxford : Oxford University Press.

Dal, G., Lignon, S., Namer, F., et Tanguy, L. (2004). « Toile contre dictionnaires : analyse morphologique en corpus de noms déverbaux concurrents ». Colloque International sur «Les noms déverbaux». Villeneuve d'Ascq : Université de Lille 3.

Dal, G., Fradin, B., Grabar, N., Namer, F., Lignon, S. et Zweigenbaum, P. (2008). «Quelques préalables au calcul de la productivité des règles constructionnelles et premiers résultats ». 1e CMLF, Paris : ILF. 1587-1599.

Dal, G. et Namer, F. (2012). «Faut-il brûler les dictionnaires ? Ou comment les ressources numériques ont révolutionné les recherches en morphologie ». $3^{\mathrm{e}}$ CMLF, Lyon : ILF. 1261-1276.

Dal, G. et Namer, F. (à paraître). « La fréquence en morphologie : pour quels usages ?». Langages.

Fradin B. (2003). Nouvelles approches en morphologie, Paris : Presses Universitaires de France.

Fradin, B., Kerleroux F. et Plénat, M. (éds) (2009) Aperçus de morphologie du français. Paris : Presses Universitaires de Vincennes.

Hathout, N. (2009). Contributions à la description de la structure morphologique du lexique et à l'approche extensive en morphologie. Mémoire d'Habilitation à Diriger des Recherches, Université de Toulouse-Le Mirail, Toulouse.

Hathout, N. (2011). «Une analyse unifiée de la préfixation en anti- ». in Boyé, G., Hathout, N., Lignon, S., Roché, M., Plénat, M., (eds.). Des Unités Morphologiques au Lexique, Paris : Hermès. 251-318. 
Hathout, N. et Namer, F. (2014). « Discrepancy between form and meaning in Word Formation: the case of over- and under-marking in French ». in Rainer, F., Dressler, W.U., Gardani, F. et Luschützky, H.C. (eds) Morphology and meaning. Amsterdam : John Benjamins. 177-190

Haspelmath, M. (2002). Understanding Morphology. London : Arnold.

Koehl, A. (2012) « Altitude, négritude, bravitude ou la résurgence d'une suffixation ». $3^{\text {e }}$ CMLF. Lyon : ILF. 1307-1323.

Labov, W. 1976 (1972). Sociolinguistique. Paris : Editions de Minuit.

Lignon, S. (2002). « L'adjectif en -ien comme révélateur de phénomènes de concurrence ». BULAG 27 : 135-150.

Lignon, S. (2008). «Les noms de spécialistes en -iste et en -ien : le chimiste perturbé ou comment le physicien se réajuste », in Vaxelaire, B., Sock, R., Kleiber, G. et Marsac F. (éds) Pertubations et Réajustement, Strasbourg : Presses Universitaires de l'Université de Marc Bloch. 287-296.

Lignon, S. (2013) « -iser and -ifier suffixation in French: verify data to verize hypotheses? » . in Hathout, N., Montermini, F., Tseng, J. (éds) Selected Proceedings of the 7th Décembrettes: Morphology in Toulouse. München : Lincom Europa. 109-132.

Lignon, S. et Namer, F. (2010). « Comment conversionner les V-ion ? ou la construction de V-ionner par conversion ». 2e CMLF. New Orleans : ILF. 1009-1028.

Lignon, S. et Namer, F. (2013). « Les créations verbales dénominales en -iser et -ifier de la Toile : concurrence morphologique et interfaces sémantico-phonologiques. » présenté à Morphologie et Interfaces, Lille.

Lignon, S. et Plénat, M. (2009), «Echangisme suffixal et contraintes phonologiques». in Fradin, B., Kerleroux, F. et Plénat, M. (éds) Aperçus de morphologie du français. Paris : Presses Universitaires de Vincennes. 65-81.

Namer F. et Villoing F. (2006), "Have cutthroats anything to do with tracheotomes? Distinctive properties of VN vs NV compounds in French". in Booij G. et alii (eds), MMM5 Proceedings, Fréjus : CNRS. 105-124.

Lin, Y., Michel, J.-B., Lieberman Aide, E., Orwant, J., Brockman, W., and Petrov, S. (2012). "Syntactic Annotations for the Google Books Ngram Corpus". Proceedings of the 50th Annual Meeting of the Association for Computational Linguistics. Jeju, Korea, 169-174.

Littré, E. (1872). Dictionnaire de la langue française. Paris : Hachette.

Namer, F. (2013). “Adjectival bases of French -aliser and -ariser verbs: syncretism or under-specification?" in Hathout, N., Montermini, F., Tseng, J. (éds). Selected Proceedings of the 7th Décembrettes: Morphology in Toulouse. München : Lincom Europa. 185-210. 
Villoing F. et Namer F. (2014), « Composition néoclassique en -logue et en -logiste : les noms en -logue sont ils encore des noms de spécialistes ? », Verbum 34(2) : 213-231.

Plag, I. (1999). Morphological productivity. Structural constraints in English derivation. Berlin/New York : Mouton de Gruyter.

Plag, I. (2003). Word-formation in English. Cambridge : Cambridge University Press.

Roché, M. (2011), «Quel traitement unifié pour les dérivations en -isme et en -iste ?», in Boyé, G., Hathout, N., Lignon, S., Roché, M., Plénat, M., (eds.). Des Unités Morphologiques au Lexique, Paris : Hermès 69-143.

Tanguy, L. (2012). Complexification des données et des techniques en linguistique : contributions du TAL aux solutions et aux problèmes. Mémoire d'Habilitation à Diriger des Recherches, Université de Toulouse-Le Mirail, Toulouse.

Villoing, F. (2012), « French compounds ». Probus, 24 (1) : 29-60. 